\title{
Influence of $\beta$-Adrenoceptor Blockade on the Myocardial Accumulation of Fatty Acid Tracer and Its Intracellular Metabolism in the Heart After Ischemia-Reperfusion Injury
}

\author{
Norio Igarashi, MD; Takashi Nozawa, MD; Nozomu Fujii, MD; \\ Takayuki Suzuki, MD; Akira Matsuki, MD; Teruo Nakadate, MD; \\ Akihiko Igawa, MD; Hiroshi Inoue, MD
}

\begin{abstract}
Background Increases in sympathetic nerve activity during ischemia may increase intracellular fatty acid (FA) accumulation via enhanced FA uptake and inhibition of $\beta$-oxidation. Therefore, the beneficial effects of $\beta$-adrenoceptor blockade on myocardial ischemic injury might result from the suppression of FA accumulation.

Methods and Results Carvedilol $(1 \mathrm{mg} / \mathrm{kg}$ ) or propranolol $(1 \mathrm{mg} / \mathrm{kg})$ was injected $10 \mathrm{~min}$ before 15 -min occlusion of coronary artery in rats. Myocardial FA accumulation and intracellular metabolites of FA tracer were determined 3 days after reperfusion using ${ }^{125}$ I- and ${ }^{131}$ I-9-metylpentadecanoic acid (9MPA). Carvedilol significantly decreased 9MPA accumulation in both the ischemic region (IR) and non-IR, as compared with vehicle, and increased its clearance. However, the non-metabolized 9MPA fraction was not different between carvedilol- and vehicle-treated rats. Consequently, the amount of non-metabolized 9MPA in the myocardium was lower in rats treated with carvedilol than in those given vehicle. These effects of carvedilol were not different from those of propranolol.

Conclusion Beta-adrenoceptor blockade did not affect a visual assessment of the autoradiographic image of 9MPA in hearts subjected to ischemia-reperfusion, but it accelerated the clearance of 9MPA in both the IR and non-IR. The administration of $\beta$-blockade before ischemia could accelerate the recovery from ischemia-reperfusion injury by inhibiting myocardial FA accumulation before $\beta$-oxidation. (Circ J 2006; 70: 1509-1514)
\end{abstract}

Key Words: $\beta$-adrenoceptor blockade; Fatty acids; Ischemia-reperfusion; Metabolism; Myocardium; Radiotracers

$\mathbf{M}$ uch evidence has demonstrated that treatment with $\beta$-adrenoceptor blockade in patients with ischemic heart disease and chronic heart failure can improve their symptoms and prognosis. The harmful effects of adrenoceptor stimulation are related to increased oxygen consumption and enhanced free fatty acid (FFA) use secondary to elevated levels of plasma FFA and to increased intramyocardial lipolysis! However, it is still unclear if $\beta$-adrenoceptor blockade has a beneficial effect on the heart subjected to ischemia-reperfusion in terms of myocardial fatty acid (FA) metabolism.

Recently, branched-chain FA analogs, such as ${ }^{123} \mathrm{I}-15-$ $\beta$-methyl-p-iodophenyl-pentadecanoic acid (BMIPP) and ${ }^{123}$ I-15-(p-iodophenyl)-9-methylpentadecanoic acid (9MPA), have been clinically applied to assess FA metabolism in patients with ischemic heart disease ${ }^{2-6}$ In contrast to a straightchain FA, methyl-branched FA analogs are expected to interfere with $\beta$-oxidation because the metabolic process requiresa-oxidation before $\beta$-oxidation? However, previous studies $^{8-10}$ demonstrated that branched-chain FA analogs

(Received June 14, 2006; revised manuscript received August 29, 2006; accepted September 4, 2006)

Second Department of Internal Medicine, Faculty of Medicine, University of Toyama, Toyama, Japan

Mailing address: Takashi Nozawa, MD, Second Department of Internal Medicine, Faculty of Medicine, University of Toyama, 2630 Sugitani, Toyama 930-0194, Japan. E-mail: tnozawa@med.u-toyama. ac.jp could be metabolized within the myocardium by $\alpha$ - and $\beta$ oxidation, and cardiac imaging using these analogs could reflect, at least in part, myocardial FA metabolism including $\beta$-oxidation. Most patients with ischemic heart disease are treated with $\beta$-adrenoceptor blockade and therefore it is important to clarify the influence of $\beta$-blockade on cardiac accumulation of the FA tracer and its intracellular metabolism. The purpose of this study was to determine the effect of carvedilol on the autoradiographic images obtained using 9MPA, as well as alterations in the intracellular oxidation of the tracer, in rat hearts after ischemia-reperfusion injury and compare it with propranolol.

\section{Methods}

\section{Experimental Animals}

All animal experiments were in accordance with the guidelines for animal experimentation and were approved by the University of Toyama.

Male Wistar rats weighing 300-350g were used for induction of ischemia-reperfusion injury as described previously $!^{11}$ Briefly, under ether anesthesia, a left thoracotomy was performed to expose the heart. The left coronary artery was ligated $2-3 \mathrm{~mm}$ from its origin with a 5-0 Prolene suture (Ethicon Inc, Somerville, NJ, USA) for $15 \mathrm{~min}$ and then the ligation was released. Sham operation was performed using the same procedure except for the coronary artery ligation. The rats were divided into 4 groups: saline 
Table 1 Plasma Concentrations of FFA, TG, and Glucose in the 4 Treatment Groups

\begin{tabular}{lcccc}
\hline \hline & $\begin{array}{l}\text { Sham } \\
(n=8)\end{array}$ & $\begin{array}{c}\text { Vehicle } \\
(n=5)\end{array}$ & $\begin{array}{c}\text { Propranolol } \\
(n=8)\end{array}$ & $\begin{array}{c}\text { Carvedilol } \\
(n=7)\end{array}$ \\
\hline FFA $(\mathrm{mmol} / \mathrm{L})$ & $1.03 \pm 0.15$ & $0.90 \pm 0.16$ & $0.89 \pm 0.20$ & $0.93 \pm 0.14$ \\
TG $(\mathrm{mg} / \mathrm{dl})$ & $31.8 \pm 9.4$ & $24.2 \pm 11.7$ & $35.5 \pm 9.8$ & $28.1 \pm 10.9$ \\
Glucose $(\mathrm{mg} / \mathrm{dl})$ & $122.5 \pm 21.4$ & $134.1 \pm 10.6$ & $120.7 \pm 18.1$ & $116.4 \pm 14.3$ \\
\hline
\end{tabular}

FFA, free fatty acids; TG, triglycerides. Mean $\pm S D$.

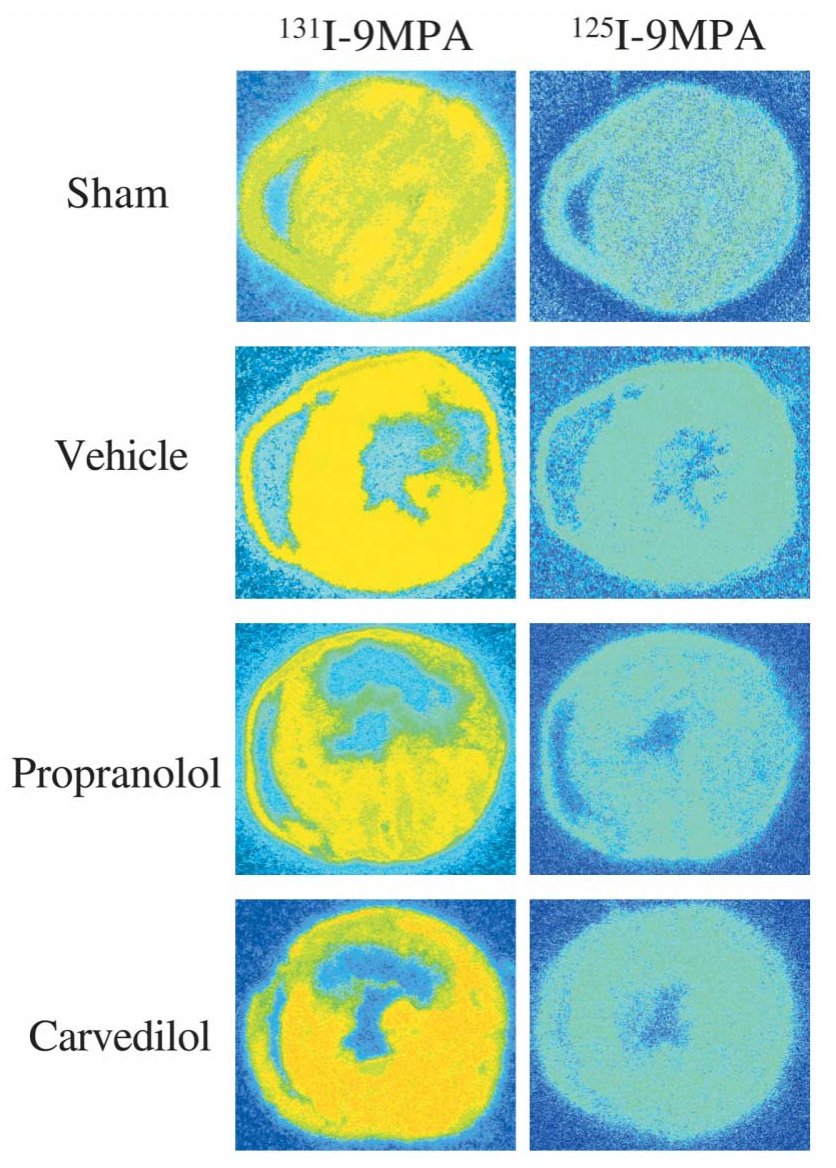

Fig 1. Representative examples of myocardial 9-methylpentadecanoic acid (9MPA) accumulation shown by dual-tracer autoradiography. ${ }^{131} \mathrm{I}-9 \mathrm{MPA}$, images obtained $5 \mathrm{~min}$ after tracer injection; ${ }^{125} \mathrm{I}$ 9MPA, $60 \mathrm{~min}$ after injection.

infusion (vehicle), carvedilol ( $1 \mathrm{mg} / \mathrm{kg}$, iv), propranolol $(1 \mathrm{mg} / \mathrm{kg}$, iv), and sham operation. Beta-adrenoceptor blockade or saline was administered intravenously 10 min before the coronary occlusion. Cardiac dual-tracer autoradiography was performed to determine myocardial accumulation and clearance of the FA analog 3 days after the ischemic insult. In separate animals, thin-layer chromatography (TLC) was performed to determine the intracellular metabolic products of the FA analog 3 days after the ischemic insult. Cardiac dual-tracer autoradiography and TLC were performed after the animals were fasted for $24 \mathrm{~h}$ before the study. Blood sampling was performed during the TLC study to determine plasma concentrations of FFA, triglycerides (TG), and glucose.

\section{FA Tracers}

In the present study, myocardial FA metabolism was

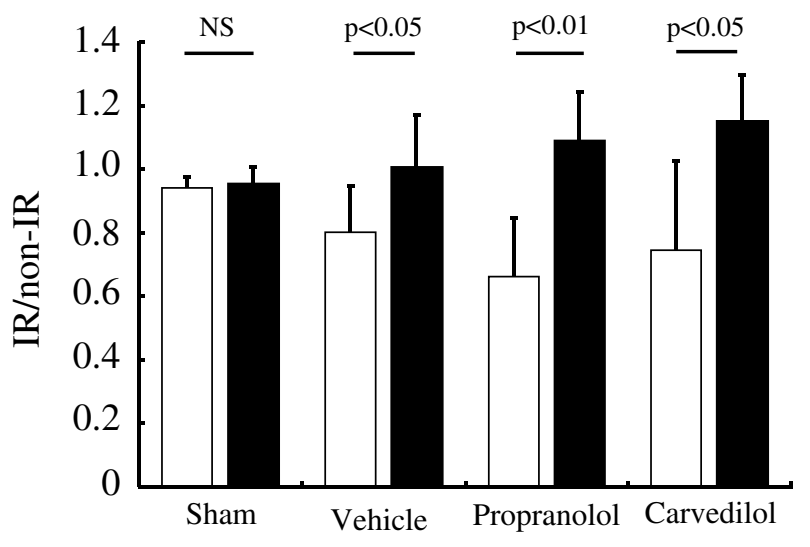

Fig 2. Nine-methylpentadecanoic acid (9MPA) accumulation ratio of the ischemic region (IR) to non-ischemic region (Non-IR) in shamoperated $(n=4)$, vehicle $(n=6)$, propranolol $(n=6)$, and carvedilol rats $(\mathrm{n}=5)$. (Open bars) ${ }^{131}$ I-9MPA accumulation ratio; (solid bars) ${ }^{125} \mathrm{I}-$ 9MPA accumulation ratio. Mean + SD.

assessed with ${ }^{131} \mathrm{I}$-and ${ }^{125} \mathrm{I}$-9MPA. 9MPA was prepared and supplied by Daiichi Radioisotope Laboratory Co, Ltd (Tokyo, Japan) and its radiochemical was more than $98 \%$, and its specific activity was $30-70 \mathrm{GBq} / \mathrm{mmol}$.

\section{Cardiac 9MPA Accumulation}

Dual-tracer autoradiography with $131 \mathrm{I}-9 \mathrm{MPA}$ and $125 \mathrm{I}-$ 9MPA was performed to determine the myocardial accumulation and clearance of 9MPA as described previously 10 Briefly, animals were injected intravenously with $0.74 \mathrm{MBq}$ of $125 \mathrm{I}-9 \mathrm{MPA}$ and $55 \mathrm{~min}$ later with $5.55 \mathrm{MBq}$ of ${ }^{131} \mathrm{I}-$ 9MPA. The hearts were removed 5 min after the second injection. Serial $20 \mu \mathrm{m}$ transverse sections were prepared. The first autoradiographic exposure on an imaging plate (BAS-UR, Fuji Film, Tokyo, Japan) was carried out for $8 \mathrm{~h}$ to reveal ${ }^{131} \mathrm{I}-9 \mathrm{MPA}$ accumulation. The second exposure for the 125 I-9MPA image was initiated 75 days later following the decay of ${ }^{131}$ I-9MPA activity, and required 30 days for adequate image quality. With the present doses of ${ }^{131} \mathrm{I}$ and ${ }^{125} \mathrm{I}$, the cross-talk between the 2 tracers was less than $3 \% 10$

To determine the myocardial accumulation of 9MPA, the autoradiographic images were analyzed using a computerassisted imaging-processing system (BAS3000, Fuji Film) as described previously 10 The region of interest was put on the left ventricular (LV) anterior wall (ischemic region, IR) and septal wall (non-ischemic region, non-IR) at the level of papillary muscles, and the IR was defined as one-sixth of the whole LV area around the center of ischemia on the autoradiographic image!1 The myocardial tracer uptake in the IR and non-IR was normalized as a percentage of the administered dose per gram of heart tissue (\%dose/g) using ${ }^{131} \mathrm{I}-$ and ${ }^{125} \mathrm{I}$-labeled graded standards. The clearance of 9MPA (ie, its washout rate (WR)), in the IR and non-IR 
Table 2 Myocardial 9MPA Accumulation and Washout Rate in the 4 Treatment Groups

\begin{tabular}{|c|c|c|c|c|c|c|}
\hline & \multicolumn{2}{|c|}{${ }^{131} I$ (\%dose/g) } & \multicolumn{2}{|c|}{${ }^{125}$ I (\%dose/g) } & \multicolumn{2}{|c|}{$W R(\%)$} \\
\hline & $I R$ & Non-IR & $I R$ & Non-IR & $I R$ & Non-IR \\
\hline $\operatorname{Sham}(n=4)$ & $6.00 \pm 1.04$ & $6.41 \pm 1.31$ & $2.85 \pm 0.49$ & $3.02 \pm 0.69$ & $52.2 \pm 6.3^{*}$ & $52.8 \pm 6.2$ \\
\hline Vehicle $(n=6)$ & $4.76 \pm 0.81^{\dagger}$ & $5.99 \pm 0.93$ & $3.02 \pm 0.41$ & $3.12 \pm 0.28$ & $33.3 \pm 5.6$ & $46.8 \pm 9.7 \neq$ \\
\hline Proranolol $(n=6)$ & $3.03 \pm 1.08 *$ & $4.52 \pm 0.59 *,+$ & $1.17 \pm 0.15 *$ & $1.10 \pm 0.22 *$ & $56.1 \pm 18.0 *$ & $75.1 \pm 7.0^{* \neq}$ \\
\hline Carvedilol $(n=5)$ & $2.78 \pm 0.48 *$ & $4.21 \pm 1.78^{*,+}$ & $1.11 \pm 0.30 *$ & $0.96 \pm 0.24 *$ & $59.6 \pm 11.7 *$ & $73.3 \pm 12.4^{*}, \dot{+}$ \\
\hline
\end{tabular}

$W R$, washout rate of 9MPA; IR, ischemic region; Non-IR, non-ischemic region. ${ }^{\dagger} p<0.01$ vs sham, ${ }^{*} p<0.01$ vs vehicle, ${ }^{\star} p<0.05$ vs IR.

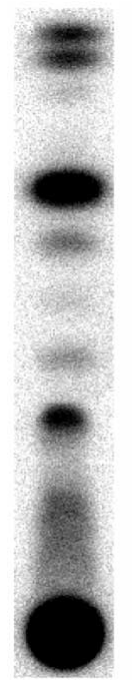

Sham
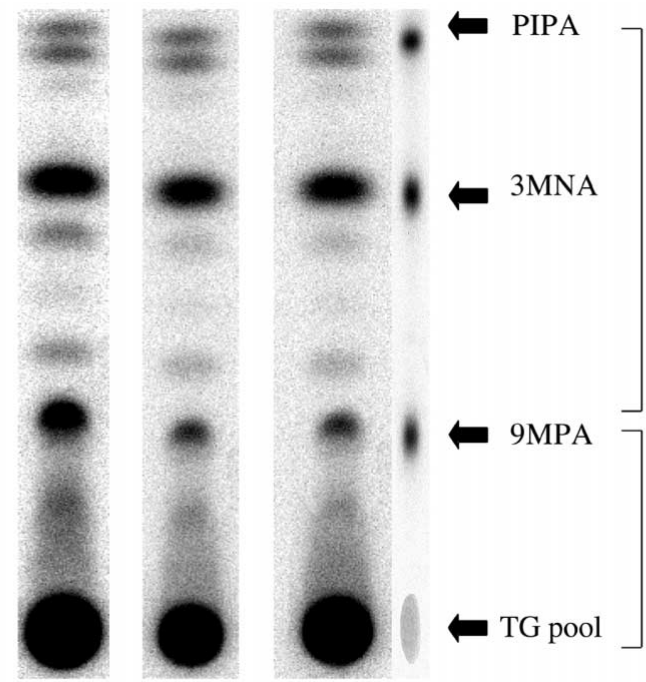

Vehicle Propranolol Carvedilol
Metabolized 9MPA fraction

Non-metabolized 9MPA fraction

was calculated using the following equation:

$$
\text { WR }(\%)=\left({ }^{131} \text { I uptake }-{ }^{125} \text { I uptake }\right) \times 100 \div{ }^{131} \text { I uptake. }
$$

\section{Analysis of 9MPA Metabolites}

Lipid extraction from myocardial tissues was performed according to a modification of the method of Folch et $\mathrm{al}^{12}$ with the metabolic products of 9MPA being assessed by TLC. A dose of $3.7 \mathrm{MBq}$ of $125 \mathrm{I}-9 \mathrm{MPA}$ was administered intravenously under pentobarbital anesthesia. The hearts were quickly removed 5 min later and the LV tissue from the IR and non-IR was separately homogenized. The radioactivity of the 9MPA metabolites was assayed by TLC on aluminum sheets (RP-18F; Merck, Germany) in conjunction with standard lipid preparations. The metabolites of 9MPA on the aluminum sheets were exposed on the imaging plate for 14 days and quantified with a bioimaging analyzer (BAS3000). Two major metabolites of 9MPA detected on the exposed images were 3-methylnonanoic acid (3MNA) as the intermediate metabolite after 3 cycles of $\beta$-oxidation from 9MPA, and p-iodo-phenyl acetic acid (PIPA) representing the final product of 9MPA $!^{3}$

\section{Statistical Analysis}

Data are expressed as mean \pm SD. Group comparisons were made using ANOVA followed by a Bonferroni test to identify differences between the groups. Comparison between the IR and non-IR in each heart was performed by paired t-tests. A value of $\mathrm{p}<0.05$ was considered statistically significant.

\section{Results}

\section{Cardiac 9MPA Accumulation}

None of the plasma levels of FFA, TG, or glucose differed among the 4 groups (Table 1 ). LV accumulation of ${ }^{131} \mathrm{I}-$ and ${ }^{125} \mathrm{I}-9 \mathrm{MPA}$ was homogeneous in the sham rats. However, in the other 3 groups (ie, vehicle, carvedilol, and propranolol groups) ${ }^{131}$ I-9MPA accumulation was lower in the IR as compared with the non-IR. In contrast, ${ }^{125}$ I-9MPA accumulation in the LV was relatively homogeneous in these groups, suggesting slower 9MPA clearance in the IR than in the non-IR (Figs 1,2). Carvedilol decreased ${ }^{131} \mathrm{I}-$ and $125 \mathrm{I}-9 \mathrm{MPA}$ accumulation in both the IR and non-IR, and the WR of the tracer was significantly greater in the carvedilol group than in the vehicle group (Table 2). None of the autoradiographic indices in the rats treated with propranolol differed from those with carvedilol.

\section{Analysis of 9MPA Metabolites}

Representative examples of TLC in the IR and non-IR are shown in Fig 3. The sum of 3MNA, PIPA, and the other intermediate metabolites processed via $\beta$-oxidation was defined as the metabolized 9MPA fraction in the present study. In rats treated with vehicle, the metabolized fraction of the IR was less than that of the non-IR, and was also less than that of the sham rats (Table 3 ). Neither carvedilol nor propranolol affected the metabolized or non-metabolized 9MPA fraction in either the IR or non-IR.

The amount of non-metabolized 9MPA in the myocardium at $5 \mathrm{~min}$ after the tracer injection was calculated from the mean values of myocardial ${ }^{131}$ I-9MPA accumulation de- 
Table 3 Metabolized and Non-Metabolized 9MPA Fractions in the IR and Non-IR of the 4 Treatment Groups

\begin{tabular}{|c|c|c|c|c|}
\hline & \multicolumn{2}{|c|}{ Non-metabolized fraction (\%) } & \multicolumn{2}{|c|}{ Metabolized fraction (\%) } \\
\hline & $I R$ & Non-IR & $I R$ & Non-IR \\
\hline $\operatorname{Sham}(n=8)$ & $40.0 \pm 6.9$ & $39.2 \pm 5.1$ & $60.0 \pm 7.2$ & $60.8 \pm 5.3$ \\
\hline Vehicle $(n=5)$ & $53.8 \pm 2.3 *$ & $46.0 \pm 6.6^{\ddagger}$ & $46.2 \pm 2.5 *$ & $54.0 \pm 7.0+$ \\
\hline Propranolol $(n=8)$ & $48.9 \pm 4.5^{*}$ & $41.0 \pm 7.1^{\ddagger}$ & $51.1 \pm 4.6^{*}$ & $59.0 \pm 7.3^{\ddagger}$ \\
\hline Carvedilol $(n=7)$ & $51.6 \pm 1.6^{*}$ & $44.8 \pm 2.0^{\ddagger}$ & $48.4 \pm 1.7 *$ & $55.2 \pm 2.1^{\ddagger}$ \\
\hline
\end{tabular}

Non-metabolized fraction, 9MPA fraction before $\beta$-oxidation; metabolized fraction, 9MPA fraction processed by $\beta$-oxidation. Other abbreviations see in Table 1.

${ }^{*} p<0.05$ vs sham, ${ }^{\ddagger} p<0.05$ vs $I R$.

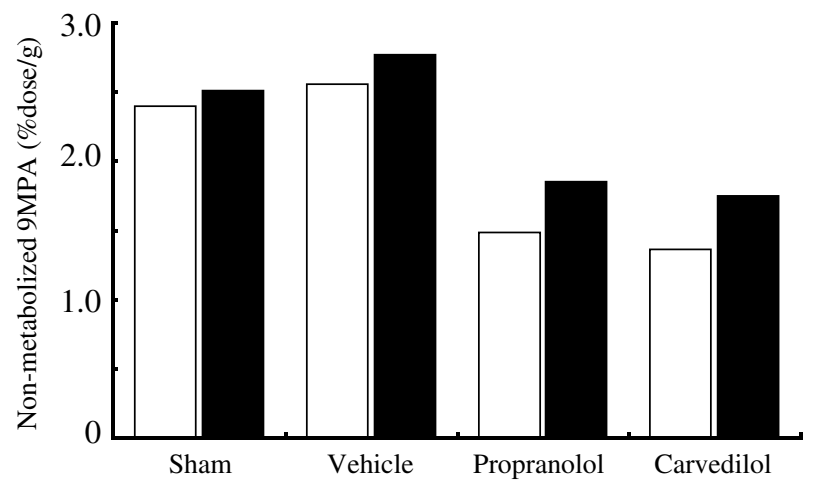

Fig 4. Amount of non-metabolized 9-methylpentadecanoic acid (9MPA) in the myocardium calculated from mean values of ${ }^{131} \mathrm{I}$ 9MPA accumulation in the ischemic (IR) and non-IR regions determined by autoradiography and the non-metabolized 9MPA fraction in the corresponding thin-layer chromatography study. (Open bars) IR; (solid bars) non-IR.

termined by dual-tracer autoradiography in each group and the non-metabolized 9MPA faction in the corresponding TLC study. As shown in Fig 4, the amount of non-metabolized 9MPA was decreased by carvedilol and propranolol in both the IR and non-IR.

\section{Discussion}

The major findings of the present study are as follows. First, a single administration of carvedilol before transient ischemia reduced myocardial 9MPA accumulation in both the IR and non-IR 3 days after reperfusion. Consequently, carvedilol did not affect a visual assessment of the autoradiographic image of 9MPA, but accelerated its clearance from both regions. Secondly carvedilol suppressed the intracellular accumulation of non-metabolized 9MPA, which implies that the administration of carvedilol before the onset of ischemia might have a beneficial effect on myocardial metabolism late in the reflow phase, because the accumulation of FA intermediates such as long-chain acyl-coenzyme A (CoA) and long-chain acylcarnitine has been implicated in the acceleration of ischemic heart failure!4-16 Finally, these effects of carvedilol were not different from those of propranolol, suggesting that the $\beta$-adrenoceptor blocking action of carvedilol might primarily contribute to alterations in cardiac FA metabolism.

\section{Effects of $\beta$-Adrenoceptor Blockade on Cardiac FA Imaging}

Branched-chain FA analogs such as 123I-BMIPP and 123I-9MPA are used clinically to assess FA metabolism in patients with coronary heart disease ${ }^{2-6}$ Slow recovery of the tracer uptake after ischemic insult has been found in both clinical and experimental studies ${ }^{17-19}$ In the present study, the reduced tracer uptake by the IR seen in the early image after tracer injection was not present in the delayed image because of slow clearance of the tracer in the IR, a finding consistent with the previous study $!^{10}$

Beta-adrenoceptor blockers, including carvedilol, are frequently prescribed for patients with coronary heart disease and heart failure, so it is important to clarify their influence on the distribution and intracellular metabolism of FA tracers in the myocardium. Miura et al reported that propranolol inhibited the myocardial accumulation of non-esterified FA in ischemic canine hearts using fluorescent labeling. ${ }^{20}$ In a study using positron emission tomography, carvedilol decreased the mean cardiac uptake rate of a long-chain FA tracer in patients with ischemic cardiomyopathy. 21 In the present study, carvedilol suppressed myocardial accumulation of 9MPA in both the IR and non-IR and consequently, the autoradiographic images from hearts treated with carvedilol were visually similar to those from the vehicle group; that is, reduced accumulation of tracer in the IR in the early image and relatively homogeneous distribution of the tracer in the delayed image. These effects of carvedilol were not different from those of propranolol, suggesting the primary influence of $\beta$-adrenoceptor blocking action of carvedilol is on tracer kinetics, but not other mechanisms, such as a-blocking or antioxidant effects, that have been implicated previously?2,23 The present results also imply that the efficacy of cardiac FA imaging for the detection of coronary heart disease would not be decreased by treatment with $\beta$-blockers.

\section{Effects of $\beta$-Blockade on Myocardial FA Metabolism After Ischemia-Reperfusion}

Metabolism of ischemic tissue is characterized by decreased oxidation of long-chain FA with increased shunting of FA to TG. Myocardial ischemia causes augmentation of intramyocardial lipolysis, as well as increasing myocardial extraction of FA from blood because of the increased plasma concentration. Increased myocardial accumulation of acyl CoA may exert several deleterious effects, including uncoupling of oxidative phosphorylation, alteration of mitochondrial permeability, and impaired sarcolemmal function and intracellular $\mathrm{Ca}^{2+}$ handling1,24 In the present study, the amount of non-metabolized 9MPA in the IR of the vehicle group did not decrease (Fig 4), despite a significant decrease in 9MPA accumulation assessed by autoradiography. These results suggest that no reduction in the level of acyl CoA in ischemia-reperfused myocardium may contribute to deterioration or slow recovery of myocardial function.

Reduction in myocardial 9MPA accumulation induced by carvedilol did not result from altered substrate availabili- 
ty, because there were no significant changes in the plasma levels of FFA and glucose among the groups in the present study. Nanki et al showed that metoprolol inhibited the accumulation of acyl $\mathrm{CoA}$ in the mitochondria of ischemic myocardium. 25 Panchal et al demonstrated a $28 \%$ decrease in the activity of myocardial carnitine palmitoyl transferase I (CPT-I), a key enzyme involved in mitochondrial FA uptake, after metoprolol treatment in dogs with coronary microembolization-induced heart failure ${ }^{26}$ Moreover, DeGrado et al have clearly shown a marked reduction in myocardial accumulation of $14(\mathrm{R}, \mathrm{S})-{ }^{18} \mathrm{~F}$-fluoro-6-thia-heptadecanoic acid in mice treated with the CPT- 1 inhibitor ${ }^{27}$ Thus, the decreased CPT-I activity by $\beta$-blockade may account for the reduction in myocardial FA accumulation, especially long-chain acyl CoA, after an ischemic insult.

\section{Propranolol vs Carvedilol}

Both propranolol and carvedilol are non-selective $\beta$ adrenoceptor blocking agents, but carvedilol has a $\mathbf{\alpha}_{1-}$ adrenoceptor blocking action and antioxidant property as well22,23 In our preliminary study using sham-operated rats, the heart rate reduction induced by the present dose of carvedilol $(1 \mathrm{mg} / \mathrm{kg})$ was similar to that of propranolol $(1 \mathrm{mg} / \mathrm{kg})(19.0 \pm 8.9 \%, \mathrm{n}=8$ vs $18.5 \pm 8.0 \%, \mathrm{n}=8)$. Therefore, the magnitude of $\beta$-blocking action of carvedilol might be equivalent to that of propranolol in the present study. The influence of carvedilol on 9MPA accumulation and its intracellular metabolism did not differ from that of propranolol. Taken together, alterations in myocardial FA metabolism by carvedilol might result primarily from its $\beta$-blocking property. In a recent study by Gambert et al ${ }^{28}$ however, trimetazidine, an inhibitor of cardiac FA oxidation, improved postischemic mechanical dysfunction in association with reduced formation of free radicals in isolated rat hearts after reperfusion following 30-min global ischemia. They suggested that adverse effects of FAs on ischemia-reperfusion injury might be mediated, at least in part, by free radicals. In the present study, 15-min coronary occlusion followed by 3-day reperfusion did not result in macroscopic myocardial infarction, although we did not determine cardiac hemodynamics or oxidative stress. It remains unclear whether the antioxidant effects of carvedilol could attenuate myocardial ischemia-reperfusion injury in the heart after more prolonged coronary occlusion.

\section{Study Limitations}

Some methodologic limitations deserve comment. First, we did not determine whether $\beta$-adrenoceptor blockade might affect cardiac FA metabolism during ischemia or during reperfusion. Many factors could contribute to myocardial reperfusion injury, but free radical generation and intracellular $\mathrm{Ca}^{2+}$ overload are considered as major factors in reperfusion injury. ${ }^{29}$ Carvediolol has an antioxidant property, but its effect on 9MPA metabolism was not different from that of propranolol in the present study. Therefore, it seems likely that the $\beta$-blocking action of these drugs might affect cardiac metabolism during ischemia rather than during reperfusion. Second, effects of $\beta$-blockade on cardiac function were not evaluated in the present study. A 15-min coronary occlusion did not result in myocardial necrosis in our previous study,1 and rats treated with and without $\beta$ blockade did not show macroscopic myocardial infarction in the present study. However, $\beta$-blockade before ischemia might accelerate the recovery from myocardial stunning. Previous studies have shown that $\beta$-blockade reduced in- farct size in the heart after more prolonged coronary occlusion30,31 Further studies are required to investigate whether changes in FA metabolism by $\beta$-blockade may accelerate recovery from myocardial stunning.

\section{Conclusions}

The present study results indicate that $\beta$-adrenoceptor blockade does not affect the visual assessment of autoradiographic images of FA tracer in the rat heart after ischemiareperfusion injury, suggesting that $\beta$-blockade does not affect the capability of FA tracer to detect coronary heart disease. Branched chain FA analogs are processed, at least in part, viaa - and $\beta$-oxidation in the in-situ heart, and, therefore a dynamic study of FA tracer accumulation, including 9MPA and BMIPP, may provide useful information on intracellular FA metabolism.

\section{References}

1. Katz AM, Messineo FC. Lipid-membrane interaction and the pathogenesis of ischemic damage in the myocardium. Circ Res 1981; 48: $1-16$.

2. Knapp FF Jr, Goodman MM, Callahan PA, Kirsch G. Radioiodinated 15-(p-iodophenyl)-3,3-dimethylpentadecanoic acid: A useful new agent to evaluate myocardial fatty acid uptake. J Nucl Med 1986; 27: $521-531$.

3. Franken RP, Geeter DF, Dendale P, Demoor D, Block P, Bossuyt A. Abnormal free fatty acid uptake in subacute myocardial infarction after coronary thrombolysis: Correlation with wall motion and inotropic reserve. J Nucl Med 1994; 35: 1758-1765.

4. Dilsizian V, Bateman TM, Bergmann SR, Prez RD, Magram MY, Goodbody AE, et al. Metabolic imaging with $\beta$-methyl- $p$-[ $\left.{ }^{123} I\right]$-iodophenyl-pentadecanoic acid identifies ischemic memory after demand ischemia. Circulation 2005; 112: 2169-2174.

5. Hashimoto J, Kubo A, Iwasaki R, Fujii H, Kunieda E, Iwanaga S, et al. Scintigraphic evaluation of myocardial ischemia using a new fatty acid analogue: Iodine-123-labelled 15-(p-iodophenyl)-9-(R,S)-methylpentadecanoic acid (9MPA). Eur J Nucl Med 1999; 26: 887-893.

6. Fujiwara S, Takeishi Y, Tojo T, Yamaoka M, Nitobe J, Takeishi K, et al. Fatty acid imaging with 123-I-15-(p- iodophenyl)-9-R,S-methylpentadecanoic acid in acute coronary syndrome. J Nucl Med 1999; 40: $1999-2006$

7. Tamaki N, Morita K, Kuge Y, Tsukamoto E. The role of fatty acids in cardiac imaging. $J$ Nucl Med 2000; 41: 1525-1534

8. Yamamichi Y, Kusuoka H, Morishita K, Shirakami Y, Kurami M, Okano K, et al. Metabolism of iodine-123-BMIPP in perfused rat hearts. J Nucl Med 1995; 36: $1043-1054$.

9. Hosokawa R, Nohara R, Fujibayashi Y, Okuda K, Ogino M, Hata T, et al. Myocardial kinetics of iodine-123-BMIPP in canine myocardium after regional ischemia and reperfusion: Implications for clinical SPECT. J Nucl Med 1997; 37: 1857-1863.

10. Igarashi N, Nozawa T, Fujii N, Kato B, Nonomura M, Matsuki A, et al. Evaluation of fatty acid metabolism in hearts after ischemiareperfusion injury using a dual isotope autoradiographic approach and tissue assay for metabolites of tracer. J Nucl Med 2005; 46: 160 164.

11. Kato B, Nozawa T, Igarashi N, Nonomura M, Fujii N, Igawa A, et al. Discrepant recovery course of sympathetic neuronal function and $\beta$ adrenoceptors in rat hearts after reperfusion following transient ischemia. J Nucl Med 2004; 45: 1074-1080.

12. Folch J, Lees M, Stanley SH. A simple method for the isolation and purification of total lipids from animal tissues. J Biol Chem 1957; 226: $497-509$.

13. Fujii N, Nozawa T, Igawa A, Kato B, Igarashi N, Nonomura M, et al. Saturated glucose uptake capacity and impaired fatty acid oxidation in hypertensive hearts before development of heart failure. Am J Physiol Heart Circ Physiol 2004; 287: H760-H766.

14. Liedtke AJ, Nellis S, Neely JR. Effects of excess free fatty acids on mechanical and metabolic function in normal and ischemic myocardium in swine. Circ Res 1978; 43: 652-661.

15. Liu Q, Docherty JC, Rendell JC, Clanachan AS, Lopaschuk GD. High levels of fatty acids delay the recovery of intracellular $\mathrm{pH}$ and cardiac efficiency in post-ischemic hearts by inhibiting glucose oxidation. J Am Coll Cardiol 2002; 39: 718-725.

16. Korge P, Honda HM, Weiss JN. Effects of fatty acids in isolated 
mitochondria: Implications for ischemic injury and cardioprotection. Am J Physiol Heart Circ Physiol 2003; 285: H259-H269.

17. Tsubokawa A, Lee JD, Shimizu H, Nakano A, Uzui H, Takeuchi M, et al. Recovery of perfusion, glucose utilization and fatty acid utilization in stunned myocardium. J Nucl Med 1997; 38: 1835-1837.

18. Buxton DB, Mody FV, Krivokapich J, Phelps ME, Schelbert HR. Quantitative assessment of prolonged metabolic abnormalities in reperfused canine myocardium. Circulation 1992; 85: 1842-1856.

19. Heyndrickx GR, Wijns W, Vogelaers D, Degrieck Y, Bol A, Vandeplasshe G, et al. Recovery of regional contractile function and oxidative metabolism in stunned myocardium induced by 1-hour circumflex coronary artery stenosis in chronically instrumented dogs. Circ Res 1993; 72: $901-913$.

20. Miura I, Hashizume H, Abiko Y. Propranolol inhibits accumulation of non-esterified fatty acids in the ischemic dog heart. Eur J Pharmacol 1988; 152: 281-288.

21. Wallhaus TR, Taylor M, DeGrado TR, Russel DC, Stanko P, Nickles RJ, et al. Myocardial free fatty acid and glucose use after carvedilol treatment in patients with congestive heart failure. Circulation 2001; 103: $2441-2446$.

22. Feuerstein GZ, Yue TL, Cheng HY, Ruffolo RR Jr. Myocardial protection by the novel vasodilating beta-blocker, carvedilol: Potential relevance of anti-oxidant activity. J Hypertens 1993; 11(Suppl): $\mathrm{S} 41-\mathrm{S} 48$.

23. Cargnoni A, Ceconi C, Bernocchi P, Boraso A, Parrinello G, Curello $\mathrm{S}$, et al. Reduction of oxidative stress by carvedilol: Role in maintenance of ischaemic myocardium viability. Cardiovasc Res 2000; 47: $556-566$.

24. Corr PB, Gross RW, Sobel BE. Amphipathic metabolites and mem- brane dysfunction in ischemic myocardium. Circ Res 1984; 55: 135 154.

25. Nanki M, Itoh K, Matsubara T, Nishimura K, Kambe T, Sugiyama S, et al. Evaluation of the effect of metoprolol on energy metabolism in the ischaemic myocardium in relation to regional myocardial blood flow. Cardiovasc Res 1987; 21: 660-667.

26. Panchal AR, Stanley WC, Kerner J, Sabbah HN. Beta-receptor blockade decreases carnitine palmitoyl transferase I activity in dogs with heart failure. J Card Fail 1998; 4: 121-126.

27. DeGrado TR, Coenen HH, Stocklin G. 14(R,S)-[18F]fluoro-6-thiaheptadecanoic acid (FTHA): Evaluation in mouse of a new probe of myocardial utilization of long chain fatty acids. J Nucl Med 1991; 32: $1888-1896$.

28. Gambert S, Vergely C, Filomenko R, Moreau D, Bettaieb A, Opie $\mathrm{LH}$, et al. Adverse effects of free fatty acid associated with increased oxidative stress in postischemic isolated rat hearts. Mol Cell Biol 2006; 283: $147-152$.

29. Opie LH. Myocardial reperfusin: New ischemic syndromes. In: Opie LH, editor. The Heart: Physiology from Cell to Circulation. Philadelphia: Lippincott-Raven; 1998; 563-588.

30. Bril A, Slivjak M, DiMartino MJ, Feuerstein GZ, Linee P, Poyser $\mathrm{RH}$, et al. Cardioprotective effects of carvedilol, a novel beta adrenoceptor antagonist with vasodilating properties, in anaesthetised minipigs: Comparison with propranolol. Cardiovasc Res 1992; 26: $518-525$.

31. Nonomura M, Nozawa T, Matsuki A, Nakadate T, Igarashi N, Kato $\mathrm{B}$, et al. Ischemia-induced norepinephrine release, but not norepinephrine-derived free radicals, contributes to myocardial ischemiareperfusion injury. Circ J 2005; 69: 590-595. 